\title{
Constraining Fundamental Physics with Future CMB Experiments
}

\author{
Silvia Galli ${ }^{a, b}$, Matteo Martinelli ${ }^{a}$, Alessandro Melchiorri ${ }^{a}$, Luca Pagano $^{a}$, Blake D. Sherwin ${ }^{c}$, David N. Spergel $^{d}$ \\ a Dipartimento di Fisica and Sezione INFN, Università di Roma "La Sapienza", Ple Aldo Moro 2, 00185, Rome, Italy \\ ${ }^{b}$ Laboratoire Astroparticule et Cosmologie (APC), \\ Université Paris Diderot, 75205 Paris cedex 13, France \\ ${ }^{c}$ Department of Physics, Princeton University, Princeton, NJ 08544-1001, USA and \\ $d$ Dept. of Astrophysical Sciences, Peyton Hall, \\ Princeton University, Princeton, NJ 08544-1001, USA
}

\begin{abstract}
The Planck experiment will soon provide a very accurate measurement of Cosmic Microwave Background anisotropies. This will let cosmologists determine most of the cosmological parameters with unprecedented accuracy. Future experiments will improve and complement the Planck data with better angular resolution and better polarization sensitivity. This unexplored region of the $\mathrm{CMB}$ power spectrum contains information on many parameters of interest, including neutrino mass, the number of relativistic particles at recombination, the primordial Helium abundance and the injection of additional ionizing photons by dark matter self-annihilation. We review the imprint of each parameter on the $\mathrm{CMB}$ and forecast the constraints achievable by future experiments by performing a Monte Carlo analysis on synthetic realizations of simulated data. We find that next generation satellite missions such as CMBPol could provide valuable constraints with a precision close to that expected in current and near future laboratory experiments. Finally, we discuss the implications of this intersection between cosmology and fundamental physics.

PACS numbers: $98.80 . \mathrm{Cq}$
\end{abstract}

\section{INTRODUCTION}

Starting with COBE's groundbreaking detection of microwave background fluctuations [1], the past two decades have seen dramatic improvements in measurements of the microwave background temperature fluctuations (see e.g. [2] and [3]). Planck's highly anticipated temperature power spectrum measurements (see [4]) will further advance this program and produce significantly improved constraints on cosmological parameters.

While Planck's measurement of the anisotropy power spectrum to multipoles $\ell \sim 2000$ will extract most of the information in primordial temperature fluctuations, ongoing and planned ground-based and balloon-based experiments are exploring two important open frontiers: (a) the measurement of extremely $\left(\leq 5^{\prime}\right)$ small-scale temperature and polarization fluctuations [5] and (b) the search for primordial B-modes, the distinctive signature of gravitational waves from inflation, on large scales [6].

For example, balloon-borne experiments such as EBEX [11] and SPIDER [12] will improve the measurements of CMB polarization while ground based telescopes such as the Atacama Cosmology Telescope (ACT) 14] and the South Pole Telescope [13] will extend temperature and polarization measurements to smaller, sub-arcminute, angular scales. Proposals for next generation CMB satellites such as CMBPol 15] or B-POL [16] are under evaluation from American and European space agencies.

What will we learn from measuring CMB temperature and polarization fluctuations on small-scales? The amplitude of temperature and polarization fluctuations is determined by several different physical effects: (1) the amplitude of primordial fluctuations, (2) the evolution of the ionization fraction of the universe at $z>1200$, which determines the sound speed for acoustic fluctuations, (3) the evolution of the ionization fraction of the universe at $z<1200$, which determines the thickness of the surface of last scatter and (4) the transition from radiation to matter domination. Moreover, while small-scale CMB fluctuations are initially pure $E$ mode, gravitational lensing rotates $E$ modes into $B$ modes $[8]$. By measuring the pattern of small scale $E$ and $B$ modes, cosmologists will be able to determine the large-scale convergence field, a direct measure of the integrated density fluctuations between redshift $z=1100$ and $z=0$ (see e.g. [22], 23]). The convergence power spectrum is particularly sensitive to density fluctuations at $z \sim 2$, an important complement to planned optical lensing measurements that probe the evolution of density fluctuations in the $z<1$ universe.

The goal of this paper is to quantify the cosmological information that could come from these new datasets. This is important for several reasons. First, while there have been many studies of the future cosmological constraints from Planck, very few papers have investigated the constraining power of combinations of future CMB datasets from different sources. Second, as we will describe in the next sections, we will consider a large set of parameters focusing on those that mainly affect the "damping tail" of the CMB angular spectrum. We consider additional parameters such as the total neutrino mass $\sum m_{\nu}$ (which affects the growth of structure in the late universe), the number of additional relativistic neutrino species $N_{\nu}^{e f f}$ (which changes the matter-radiation epoch), and possible changes in the recombination process due to changes in the fractional helium abundance $Y_{p}$, dark matter self-annihilation processes, and variations in fundamental constants such as as the fine struc- 
ture constant $\alpha$ and Newton's gravitational constant $G$. We will not only show the constraints on each single parameter but also the degeneracies among them.

We will consider 3 experimental configurations: the Planck satellite [4], the combination of Planck with ACT fitted with polarization-sensitive detectors, ACTPol, 14] and, finally, the next CMBPol satellite [15].

Recent studies have already fully demonstrated the ability of next generation satellite missions to constrain inflationary parameters [18] and the reionization history [19] in the framework of the CMBPol concept mission study (see also [20]). For this reason we will not consider primordial gravitational waves, more general reionization scenarios or experiments that will mainly probe large angular scale polarization in this paper.

This paper will show that next generation CMB experiments can significantly improve constraints on cosmology and fundamental physics and could produce a detection of neutrino mass. The paper is structured as follows. Section II describes our analysis approach. Section III presents our analysis for improved constraints from the planned ACTPol experiment and for the proposed CMBPol experiment. In Section IV we present our conclusions.

\section{FORECAST METHOD AND ASSUMPTIONS}

We generate synthetic datasets for the Planck, ACTPol and CMBPol experiments following the commonly used approach described for example in [17] and 24]. These datasets are generated starting from the assumption of a fiducial "true" cosmological model compatible with the WMAP-5 maximum likelihood parameters [60], i.e. with baryon density $\Omega_{b} h^{2}=0.0227$, cold dark matter density $\Omega_{c} h^{2}=0.110$, spectral index $n_{s}=0.963$, and optical depth $\tau=0.09$. This model also assumes a flat universe with a cosmological constant, massless neutrinos with effective number $N_{\nu}^{\text {eff }}=3.04$, standard recombination, Helium fraction $Y_{p}=0.24$ and all fundamental constants fixed to their current values (we will vary all these parameters later). Given the fiducial model, we use the publicly available Boltzmann code CAMB! 21] to calculate the corresponding theoretical angular power spectra $C_{\ell}^{T T}, C_{\ell}^{T E}, C_{\ell}^{E E}$ for temperature, cross temperaturepolarization and polarization. 2 .

The synthetic datasets are then generated by considering for each $C_{\ell}$ a noise spectrum given by:

$$
N_{\ell}=w^{-1} \exp \left(\ell(\ell+1) 8 \ln 2 / \theta^{2}\right)
$$

\footnotetext{
1 http://camb.info/

2 Note that we don't consider the $B$ mode lensing channel, we will discuss this choice later in this section
}

where $\theta$ is the FWHM of the beam assuming a Gaussian profile and where $w^{-1}$ is the experimental power noise related to the detectors sensitivity $\sigma$ by $w^{-1}=(\theta \sigma)^{2}$.

We assume that beam uncertainties are small and that uncertainties due to foreground removal are smaller than statistical errors. These are demanding assumptions; however, the experimental groups are working hard to achieve these goals.

Together with the primary anisotropy signal we also take into account information from CMB weak lensing, considering the power spectrum of the deflection field $C_{\ell}^{d d}$ and its cross correlation with temperature maps $C_{\ell}^{T d}$. A large number of methods have been suggested for lensing extraction from CMB maps. All these methods exploit the non-gaussian signal induced by lensing. Here we use the quadratic estimator method of $\mathrm{Hu} \&$ Okamoto [23], that provides an algorithm for estimating the corresponding noise spectrum $N_{\ell}^{d d}$ from the observed CMB primary anisotropy and noise power spectra.

This method doesn't include the polarization $B B$ channel since is dominated by the lensing signal. The Planck experiment is not sensitive to the $B B$ lensing signal, while the CMBPol experiment and, possibly, ACTpol could detect it. While algorithms are available that can in principle include in the forecast the lensing $B B$ signal, here we take the conservative approach to not include it. This leaves open the possibility to use this channel for further checks for foregrounds contamination and systematics.

We generate mock datasets with noise properties consistent respectively with the Planck mission (see [4]), the ACT telescope [14] and the future CMBPol experiment [15]. For the simulated Planck dataset we consider the detectors at 70,100 , and $143 \mathrm{GHz}$ while for ACTPol we use the single $150 \mathrm{GHz}$ channel. For $\mathrm{CMBPol}$ we also consider the single $150 \mathrm{GHz}$ channel. The experimental specifications are reported in Table \where the sensitivity $\sigma$ is in units of $\Delta T / T$.

Once a mock dataset is produced we compare a generic theoretical model through a likelihood $\mathcal{L}$ defined as

$$
-2 \ln \mathcal{L}=\sum_{l}(2 l+1) f_{\text {sky }}\left(\frac{D}{|\bar{C}|}+\ln \frac{|\bar{C}|}{|\hat{C}|}-3\right),
$$

where $D$ is defined as

$$
\begin{aligned}
D= & \hat{C}_{\ell}^{T T} \bar{C}_{\ell}^{E E} \bar{C}_{V}^{d d}+\bar{C}_{\ell}^{T T} \hat{C}_{\ell}^{E E} \bar{C}_{\ell}^{d d}+\bar{C}_{\ell}^{T T} \bar{C}_{\ell}^{E E} \hat{C}_{\ell}^{d d} \\
& -\bar{C}_{\ell}^{T E}\left(\bar{C}_{\ell}^{T E} \hat{C}_{\ell}^{d d}+2 \hat{C}_{\ell}^{T E} \bar{C}_{\ell}^{d d}\right) \\
& -\bar{C}_{\ell}^{T d}\left(\bar{C}_{\ell}^{T d} \hat{C}_{\ell}^{E E}+2 \hat{C}_{\ell}^{T d} \bar{C}_{\ell}^{E E}\right),
\end{aligned}
$$

where $\bar{C}_{l}$ and $\hat{C}_{l}$ are the fiducial and theoretical spectra plus noise respectively, and $|\bar{C}|,|\hat{C}|$ denote the determinants of the theoretical and observed data covariance matrices respectively, 


$$
\begin{aligned}
& |\bar{C}|=\bar{C}_{\ell}^{T T} \bar{C}_{\ell}^{E E} \bar{C}_{\ell}^{d d}-\left(\bar{C}_{\ell}^{T E}\right)^{2} \bar{C}_{\ell}^{d d}-\left(\bar{C}_{\ell}^{T d}\right)^{2} \bar{C}_{\ell}^{E E},(4) \\
& |\hat{C}|=\hat{C}_{\ell}^{T T} \hat{C}_{\ell}^{E E} \hat{C}_{\ell}^{d d}-\left(\hat{C}_{\ell}^{T E}\right)^{2} \hat{C}_{\ell}^{d d}-\left(\hat{C}_{\ell}^{T d}\right)^{2} \hat{C}_{\ell}^{E E}(5)
\end{aligned}
$$

and finally $f_{s k y}$ is the sky fraction sampled by the experiment after foregrounds removal.

We derive constraints from simulated data using a modified version of the publicly available Markov Chain Monte Carlo package cosmomc [65] with a convergence diagnostic based on the Gelman and Rubin statistic performed on 8 chains. We sample the following ninedimensional set of cosmological parameters, adopting flat priors on them: the physical baryon and Cold Dark Matter density fractions, $\omega_{b}=\Omega_{b} h^{2}$ and $\omega_{c}=\Omega_{c} h^{2}$, the ratio of the sound horizon to the angular diameter distance at decoupling, $\theta_{S}$, the scalar spectral index $n_{S}$, the overall normalization of the spectrum $A_{s}$ at $k=0.002 \mathrm{Mpc}^{-1}$, the optical depth to reionization, $\tau$, the total mass of neutrinos, $\sum m_{\nu}$, the primordial helium abundance, $Y_{p}$, and the dark energy equation of state $w$. We also consider parameters that can change the process of recombination: the dark matter selfannihilation rate $p_{a n n}$, a variation in the fine structure constant $\alpha / \alpha_{0}$ and in Newton's constant $\lambda_{G}=G / G_{0}$, where $\alpha_{0}$ and $G_{0}$ are the currently measured values. For these latter parameters we choose to sample the Hubble constant $H_{0}$ instead of $\theta_{S}$ since these parameters are derived assuming standard recombination. We also use a cosmic age top-hat prior with $10 \mathrm{Gyr} \leq t_{0} \leq 20 \mathrm{Gyr}$. Furthermore, we consider adiabatic initial conditions and we impose flatness.

In what follows we will consider temperature and polarization power spectrum data up to $\ell_{\max }=2500$, due to possible unresolved foreground contamination at smaller angular scales and larger multipoles. Measurements of small-scale temperature fluctuations by ACT 9 ] and SPT [10] confirm that extragalactic foregrounds will limit precision measurements of primordial temperature fluctuations to $\ell<2500$. Even if these foregrounds could be removed, kinetic Sunyaev-Zel'dovich (KSZ) fluctuations would provide a limiting source of confusion that will be difficult to model and impossible to remove as it has the same spectral shape as primordial fluctuations. Small-scale polarization measurements offer our best hope to probe the early universe on angular scales of $\ell=2000-4000$ : dusty galaxies are thought to be only $1-2 \%$ polarized [7]. We expect that secondary fluctuations should produce minimal polarization fluctuations and therefore that polarization will provide an unbeatable test for systematics. We have checked that including only polarization data from $\ell=2500$ up to $\ell_{\max }=3500$ does not significantly change the cosmological results, again suggesting the use of high $\ell$ polarized data for checks for systematics.

\begin{tabular}{rcccc} 
Experiment Channel & FWHM & $\Delta T / T$ & $\Delta P / T$ \\
\hline Planck & 70 & $14^{\prime}$ & 4.7 & 6.7 \\
$f_{s k y}=0.85$ & 100 & $10^{\prime}$ & 2.5 & 4.0 \\
& 143 & $7.1^{\prime}$ & 2.2 & 4.2 \\
\hline ACTPol & 150 & $1.4^{\prime}$ & 14.6 & 20.4 \\
$f_{s k y}=0.19$ & & & & \\
\hline CMBPol & 150 & 5.6 & 0.037 & 0.052 \\
$f_{s k y}=0.72$ & & & & \\
\hline
\end{tabular}

TABLE I. Planck [4], ACTPol[14] and CMBPol[15] experimental specifications. Channel frequency is given in $\mathrm{GHz}$, FWHM in arcminutes and noise per pixel in $10^{-6}$ for the Stokes I, Q and U parameters. In the analysis, we assume that beam uncertainties and foreground uncertainties are smaller than the statistical errors in each of the experiments.

\section{RESULTS}

\section{A. Constraints on the "standard" 6 parameters $\Lambda-\mathrm{CDM}$ scenario}

\begin{tabular}{r|c|cc|cc}
\hline \hline $\begin{array}{r}\text { Parameter } \\
\text { uncertainty }\end{array}$ & Planck & \multicolumn{2}{|c|}{ Planck+ACTPol } & \multicolumn{2}{|c}{ CMBPol } \\
\hline$\sigma\left(\Omega_{b} h^{2}\right)$ & 0.00013 & 0.000078 & $(1.7)$ & 0.000034 & $(3.8)$ \\
$\sigma\left(\Omega_{c} h^{2}\right)$ & 0.0010 & 0.00064 & $(1.6)$ & 0.00027 & $(3.7)$ \\
$\sigma\left(\theta_{s}\right)$ & 0.00026 & 0.00016 & $(1.6)$ & 0.000052 & $(5.0)$ \\
$\sigma(\tau)$ & 0.0042 & 0.0034 & $(1.2)$ & 0.0022 & $(1.9)$ \\
$\sigma\left(n_{s}\right)$ & 0.0031 & 0.0021 & $(1.5)$ & 0.0014 & $(2.2)$ \\
$\sigma\left(\log \left[10^{10} A s\right]\right)$ & 0.013 & 0.0086 & $(1.5)$ & 0.0055 & $(2.4)$ \\
$\sigma\left(H_{0}\right)$ & 0.53 & 0.30 & $(1.8)$ & 0.12 & $(4.4)$ \\
\hline \hline
\end{tabular}

TABLE II. $68 \%$ c.l. errors on cosmological parameters from future surveys. A "standard", 6 parameters $\Lambda$-CDM scenario is assumed. The numbers in brackets show the improvement factor $i=\sigma_{\text {Planck }} / \sigma$ respect to the Planck experiment.

In Table $\llbracket$ we report the future constraints on the parameters of a "minimal" cosmological model. Together with the standard deviations on each parameter we also report, for $\mathrm{ACTPol}$ and $\mathrm{CMBPol}$, the improvement factor for each parameter defined as the ratio $\sigma_{\text {Planck }} / \sigma$ where $\sigma$ is the error from Planck+ACTPol or CMBPol and $\sigma_{\text {Planck }}$ is the constraint from Planck.

As we can see in the Table, the combination of Planck with ACTPol will improve by a factor $\sim 1.5$ the constraints on most of the parameters derived from Planck alone. CMBPol will improve by a factor $\sim 4$ the constraints on the baryon density, $H_{0}$ and $\theta_{s}$, while the constraints on parameters as $n_{s}$ and $\tau$ are improved by a factor $\sim 2$. 


\section{B. Future Constraints on Neutrino Masses}

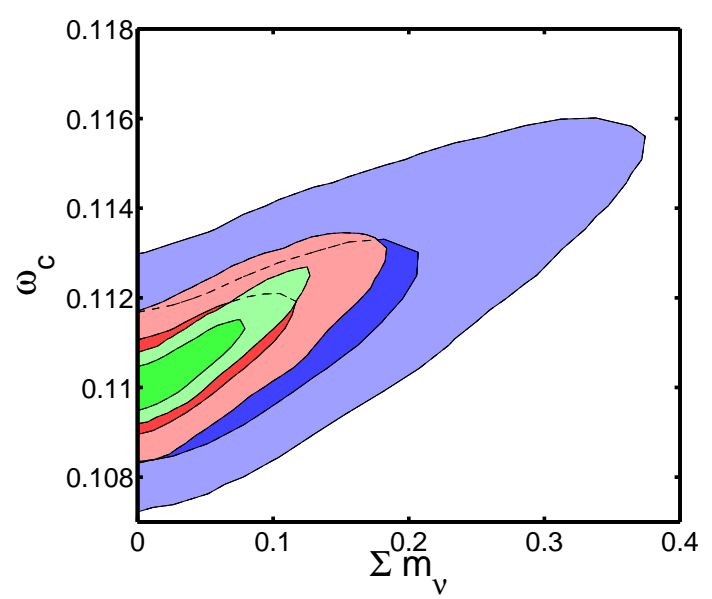

FIG. 1. $68 \%$ and $95 \%$ likelihood contour plots on the $\sum m_{\nu}$ - $\omega_{c}$ plane for Planck (blue), Planck+ACTPol (red) and CMBPol (green).

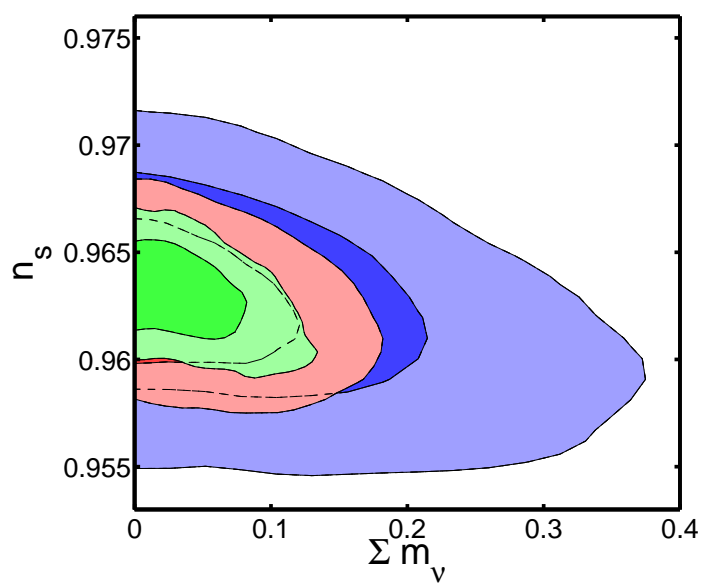

FIG. 2. $68 \%$ and $95 \%$ likelihood contour plots on the $\sum m_{\nu}$ - $n_{s}$ plane for Planck (blue), Planck+ACTPol (red) and CMBPol (green).

The detection of the absolute mass scale of the neutrino is one of the major goals of experimental particle physics. However, cosmology could provide an earlier, albeit model-dependent, detection. CMB power spectra are sensitive to a total variation in neutrino mass eigenstates $\Sigma m_{\nu}$ (see e.g. [25, 26]) but can't discriminate between the mass of a single neutrino flavour (see e.g. 27]) because of degeneracies with other parameters. Inclusion of massive neutrinos increases the anisotropy at small scales because the decreased perturbation growth contributes to the photon energy density fluctuation. Moreover, gravitational lensing leads to smoothing of the acoustic peaks and enhancement of power on the damping tail of the power spectrum; the amount of lensing is also connected to the neutrino mass (see e.g.[28]).

\begin{tabular}{r|c|cc|cc}
\hline \hline $\begin{array}{r}\text { Parameter } \\
\text { uncertainty }\end{array}$ & Planck & \multicolumn{2}{|c|}{ Planck+ACTPol } & \multicolumn{2}{|c}{ CMBPol } \\
\hline$\sigma\left(\Omega_{b} h^{2}\right)$ & 0.00014 & 0.000081 & $(1.7)$ & 0.000033 & $(4.2)$ \\
$\sigma\left(\Omega_{c} h^{2}\right)$ & 0.0017 & 0.0010 & $(1.7)$ & 0.00071 & $(2.4)$ \\
$\sigma\left(\theta_{S}\right)$ & 0.00028 & 0.00016 & $(1.7)$ & 0.000062 & $(4.5)$ \\
$\sigma(\tau)$ & 0.0042 & 0.0034 & $(1.2)$ & 0.0023 & $(1.8)$ \\
$\sigma\left(n_{S}\right)$ & 0.0034 & 0.0022 & $(1.5)$ & 0.0016 & $(2.1)$ \\
$\sigma\left(\log \left[10^{10} A_{S}\right]\right)$ & 0.013 & 0.0094 & $(1.4)$ & 0.0065 & $(2.0)$ \\
$\sigma\left(\sum m_{\nu}\right)$ & $<0.16$ & $<0.08$ & $(2.0)$ & $<0.05$ & $(3.2)$ \\
\hline \hline
\end{tabular}

TABLE III. $68 \%$ c.l. errors on cosmological parameters in the case of massive neutrinos. The numbers in brackets show the improvement factor $\sigma_{\text {Planck }} / \sigma$ respect to the Planck experiment.

Current oscillation experiments provide essentially two mass differences for the neutrino mass eigenstates: $\Delta m_{\text {solar }}^{2} \sim 8 \times 10^{-5} \mathrm{eV}^{2}$ and $\Delta m_{\text {atm }}^{2} \sim 2.5 \times 10^{-3} \mathrm{eV}^{2}$ (see e.g. [29] and references therein). An inverted hierarchy in the neutrino mass eigenstates predicts a lower limit to the total neutrino mass of about $\sum m_{\nu} \geq 0.10 \mathrm{eV}$ while a direct hierarchy predicts $\sum m_{\nu} \geq 0.05 \mathrm{eV}$. The goal for CMB experiments is therefore to have a sensitivity better than $\sum m_{\nu} \leq 0.10 \mathrm{eV}$ for possibly ruling out the inverted hierarchy and better than $\sum m_{\nu} \leq 0.05 \mathrm{eV}$ for a definitive detection of neutrino mass. As we can see from Table III the expected sensitivity from Planck and Planck+ACTPol is sufficient to find the neutrino mass in the inverted hierarchy case, while CMBPol could possibly also measure it in the direct hierarchy case. In particular, the combination of ACTPol data with Planck is expected to improve the bound on the neutrino mass by a factor of 2 while CMBPol can improve it by a factor of more than 3. These limits are far better than those expected from future laboratory experiments. The expected upper limit expected from the KATRIN [30] beta decay experiment is $m_{\nu_{e}}<0.2 \mathrm{eV}$ at $90 \%$ c.l., which roughly translates to an upper limit of $\sum m_{\nu}<0.48 \mathrm{eV}$ at one standard deviation (see [31]). Planck and Planck+ACTPol will explore the same energy scale, providing a great opportunity for confirming or anticipating a mass detection from KATRIN. Planck alone will also falsify or confirm the claim of detection of the absolute scale of the neutrino mass from the Heidelberg-Moscow neutrinoless double beta decay experiment with a effective electron neutrino mass in the range $0.2 e V<m_{\nu_{e}}<0.6 e V$ at $99.73 \%$ c.l. [32].

Future double beta decay experiments such as MARE [33] should sample mass scales of the order of $m_{\nu_{e}} \sim$ $0.2 \mathrm{eV}$. These experiments, if combined with Planck and Planck+ACTPol constraints could provide extremely valuable information on neutrino physics. For example, a CMB detection of a neutrino mass not confirmed by double beta decay experiments would rule out neutrinos as majorana-like particles.

Including a neutrino mass in the determination of the 
cold dark matter density $\omega_{c}$ results in an uncertainty that is nearly doubled with respect to the standard analysis, as we can see by comparing Table III with Table II Moreover, the constraints on $n_{s}$ are also affected. We show in Figure 1 and Figure 2 the 2-D likelihood contour plots at $68 \%$ and $95 \%$ confidence level in the $\Sigma m_{\nu}$ vs $\omega_{c}$ and vs $n_{s}$ planes respectively. As we can see, a non negligible neutrino mass has positive correlation with higher values of the cold dark matter abundance and lower values of the scalar spectral index.

\section{Future Constraints on Extra Background of Relativistic Particles}

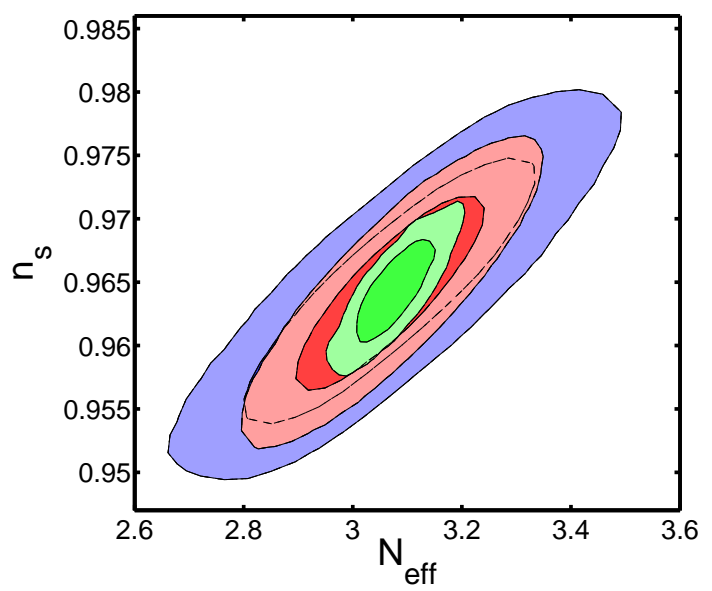

FIG. 3. $68 \%$ and $95 \%$ likelihood contour plots on the $N_{\text {eff }}$ - $n_{s}$ plane for Planck (blue), Planck+ACTPol (red) and CMBPol (green).

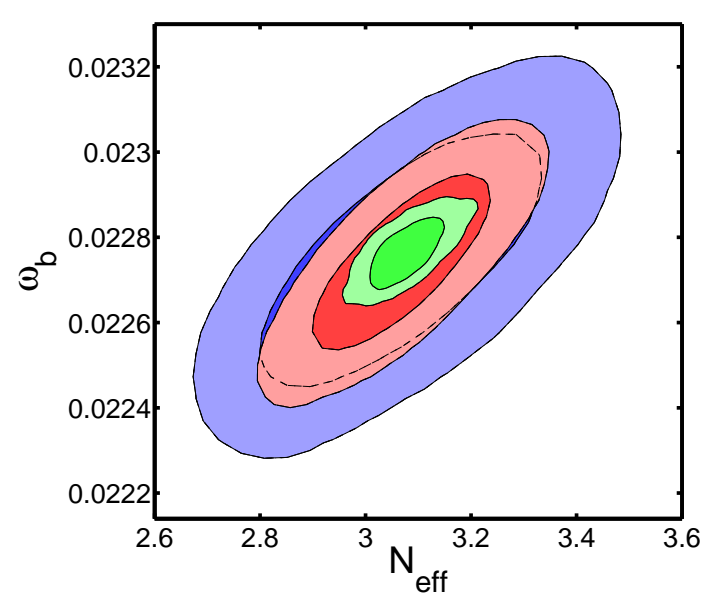

FIG. 4. $68 \%$ and $95 \%$ likelihood contour plots on the $N_{\text {eff }}$ - $\omega_{b}$ plane for Planck (blue), Planck+ACTPol (red) and CMBPol (green).

An additional background of relativistic (and noninteracting) particles can be parametrized by introducing an effective number of neutrino species $N_{\nu}^{e f f}$. This

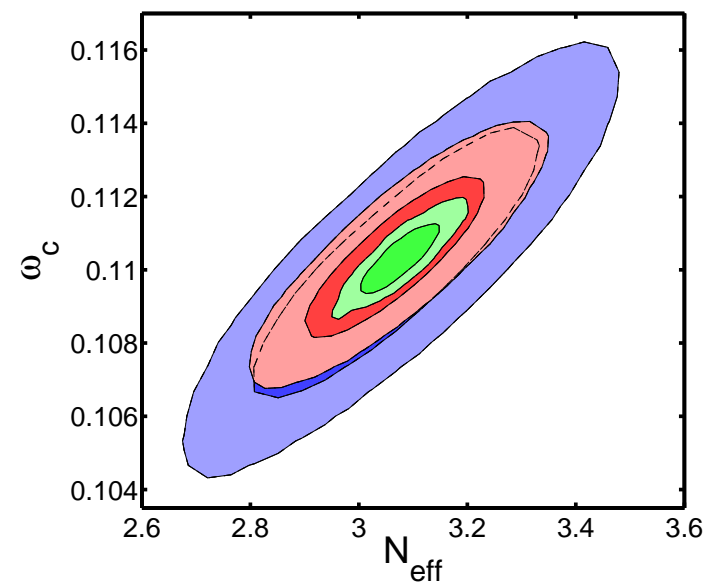

FIG. 5. $68 \%$ and $95 \%$ likelihood contour plots on the $N_{\text {eff }}$ - $\omega_{c}$ plane for Planck (blue), Planck+ACTPol (red) and CMBPol (green).

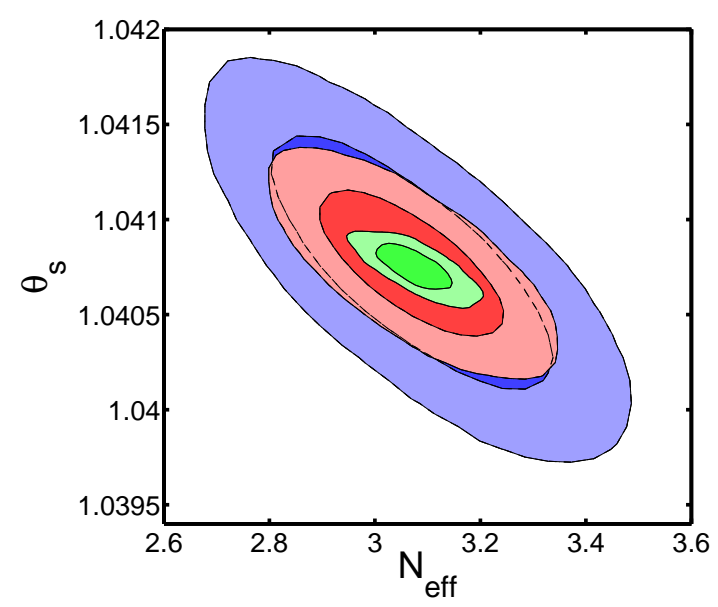

FIG. 6. $68 \%$ and $95 \%$ likelihood contour plots on the $N_{\text {eff }}-\theta_{s}$ plane for Planck (blue), Planck+ACTPol (red) and CMBPol (green).

additional background changes the $\mathrm{CMB}$ anisotropies through time variations of the gravitational potential at recombination due to the presence of this non-negligible relativistic component (the so-called early Integrated Sachs Wolfe effect). The main consequence is an increase in the small-scale CMB anisotropy (see e.g. 34]). The results are reported in Table IV.

As we can see, combining ACTPol with Planck will improve the constraint on $N_{\text {eff }}$ by a factor of 1.5 while CMBPol could improve it by a factor of $\sim 3.7$. Comparing with the results in Table III the inclusion of a background of relativistic particles strongly weakens the constraints on $n_{s}, \omega_{b}, \omega_{c}$ and $\theta_{s}$. As we can see from Figures 3 , 4, and 5 and 6 there is indeed a strong positive correlation between $N_{\nu}^{e f f}$ and these parameters (negative for $\theta_{s}$ ).

While adding ACT data will improve the constraints by a factor $\sim 2, \mathrm{CMBPol}$ can provide constraints that 


\begin{tabular}{r|c|cc|cc}
\hline \hline $\begin{array}{r}\text { Parameter } \\
\text { uncertainty }\end{array}$ & Planck & Planck+ACTPol & \multicolumn{2}{|c}{ CMBPol } \\
\hline$\sigma\left(\Omega_{b} h^{2}\right)$ & 0.00020 & 0.00013 & $(1.5)$ & 0.000048 & $(4.1)$ \\
$\sigma\left(\Omega_{c} h^{2}\right)$ & 0.0025 & 0.0015 & $(1.7)$ & 0.00058 & $(4.3)$ \\
$\sigma\left(\theta_{s}\right)$ & 0.00044 & 0.00024 & $(1.8)$ & 0.000075 & $(5.9)$ \\
$\sigma(\tau)$ & 0.0043 & 0.0035 & $(1.2)$ & 0.0023 & $(1.9)$ \\
$\sigma\left(n_{s}\right)$ & 0.0073 & 0.0049 & $(1.5)$ & 0.0026 & $(2.8)$ \\
$\sigma\left(\log \left[10^{10} A_{s}\right]\right)$ & 0.019 & 0.013 & $(1.5)$ & 0.0078 & $(2.4)$ \\
$\sigma\left(N_{e f f}\right)$ & 0.18 & 0.11 & $(1.6)$ & 0.044 & $(4.1)$ \\
\hline \hline
\end{tabular}

TABLE IV. $68 \%$ c.l. errors on cosmological parameters in the case of extra background of relativistic particles $N_{e f f}$. The numbers in brackets show the improvement factor $\sigma_{\text {Planck }} / \sigma$ respect to the Planck experiment.

could give valuable information on the physics of neutrino decoupling from the photon-baryon primordial plasma. As it is well known, the standard value of neutrino parameters $N_{\text {eff }}=3$ should be increased to $N_{\text {eff }}=3.04$ due to an additional contribution from a partial heating of neutrinos during the electron-positron annihilations (see e.g. 35]). This effect, expected from standard physics, could be tested by the CMBPol experiment, albeit at just one standard deviation. However, the presence of non standard neutrino-electron interactions (NSI) may enhance the entropy transfer from electron-positron pairs into neutrinos instead of photons, up to a value of $N_{\text {eff }}=3.12([36])$. This value could be discriminated by CMBPol from $N_{\text {eff }}=3$ at $\sim 3$ standard deviations, shedding new light on NSI models.

\section{Future Constraints on Dark Matter Self Annihilation}

Annihilating particles affect the ionization history of the universe in three different ways: the interaction of the shower produced by the annihilation with the thermal gas can ionize the gas, induce Ly- $\alpha$ excitation of the hydrogen and heat the plasma. The first two modify the evolution of the free electron fraction $x_{e}$, the third affects the temperature of baryons ([37], [38], [39] ).

The rate of energy release $\frac{d E}{d t}$ per unit volume by a relic self-annihilating dark matter particle is given by

$$
\begin{aligned}
\frac{d E}{d t}(z) & =\rho_{c}^{2} c^{2} \Omega_{D M}^{2}(1+z)^{6} p_{a n n} \\
p_{a n n} & =f \frac{<\sigma v>}{m_{\chi}}
\end{aligned}
$$

where $n_{\mathrm{DM}}(z)$ is the relic DM abundance at a given redshift $z,\langle\sigma v\rangle$ is the effective self-annihilation rate and $m_{\chi}$ the mass of our dark matter particle, $\Omega_{\mathrm{DM}}$ is the dark matter density parameter and $\rho_{c}$ is the critical density of the universe today; the parameter $f$ indicates the fraction of energy which is absorbed overall by the gas, under the approximation that the energy absorption takes place locally. The CMB is sensitive to the combined parameter $p_{a n n}$ only. The greater $p_{a n n}$, the higher the fraction of free electrons surviving after recombination, which widens the peak of the visibility function and dampens the peaks of the temperature and polarization angular power spectra.

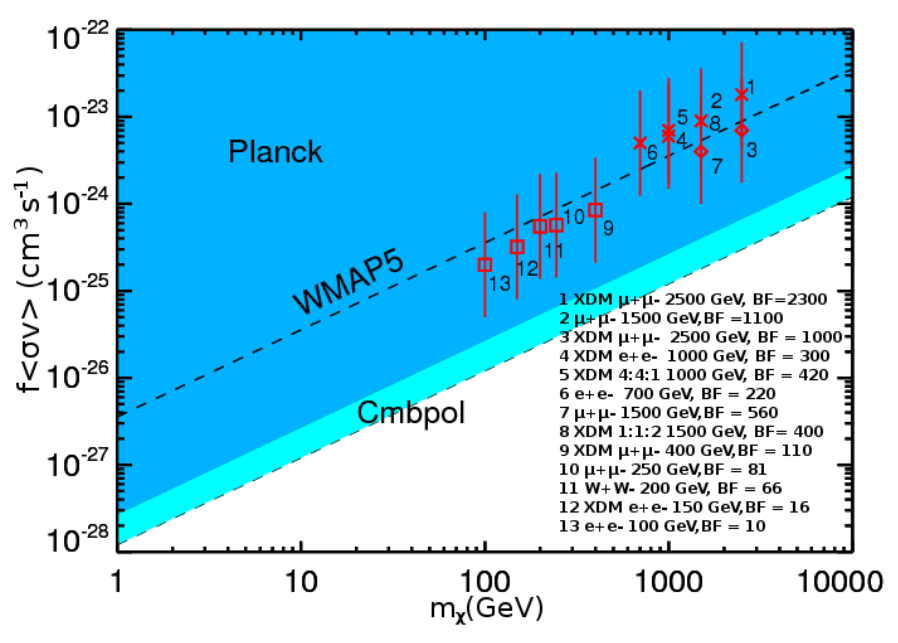

FIG. 7. Constraints on the self-annihilation cross-section at recombination $(\langle\sigma v\rangle)_{z r}$ times the gas-shower coupling parameter $\mathrm{f}$. The dark blue area is excluded by Planck at 95\% c.l., whereas the lightest blue area indicates the additional parameter space excluded by CMBpol. Plank+ACT constraint is not shown as it is only $20 \%$ tighter than the Planck constraint. The dashed line represents the current constraints given by the WMAP5 data [37]. The red data points are taken from [40] (based on the results of [44] and [45]), and indicate the positions of models of dark matter particles that fit the observed cosmic-ray excesses for PAMELA data (squares), PAMELA+FERMI (diamods) and PAMELA+ATIC (crosses). The ratios appearing in the legend indicate models of particles that annihilate through an intermediate light state to electrons, muons and pions in the given ratio. Error bars indicate astrophysical uncertainties in the cross-section boost factor. We refer to [40] for further details on these models.

As we can see by comparing the entries in Table $\nabla$ with the results in Table II the inclusion of dark matter selfannihilation doesn't substantially affect the constraints on the other parameters.

As shown in Galli et al. [37], WMAP5 data already puts interesting constraints on dark matter annihilation, namely $p_{a n n}=2.4 \times 10^{-6}\left[\mathrm{~m}^{3} / \mathrm{s} / \mathrm{Kg}\right]$ at $95 \%$ c.l.. This result disfavours dark matter annihilation as the main cause of the anomalies in the cosmic ray positron to electron fraction measured by PAMELA [41] and in the energy spectrum of cosmic ray electrons measured by ATIC [42] and less evidently by FERMI [43]. Slatyer et al. [40] examined the constraining power of this result on WIMPlike dark matter models that fit the excesses in the data. In these models, particles annihilate in leptons and pions both directly and through a new $\mathrm{GeV}$-scale state. They showed that most of these models are excluded by 


\begin{tabular}{r|c|cc|cc}
\hline \hline $\begin{array}{r}\text { Parameter } \\
\text { uncertainty }\end{array}$ & Planck & \multicolumn{2}{|l|}{ Planck+ACTPol } & CMBPol \\
\hline$\sigma\left(\Omega_{b} h^{2}\right)$ & 0.00013 & 0.000079 & $(1.6)$ & 0.000032 & $(4.1)$ \\
$\sigma\left(\Omega_{c} h^{2}\right)$ & 0.0010 & 0.00063 & $(1.6)$ & 0.00027 & $(3.7)$ \\
$\sigma\left(H_{0}\right)$ & 0.52 & 0.30 & $(1.7)$ & 0.12 & $(4.3)$ \\
$\sigma(\tau)$ & 0.0042 & 0.0034 & $(1.2)$ & 0.0023 & $(1.8)$ \\
$\sigma\left(n_{S}\right)$ & 0.0032 & 0.0021 & $(1.5)$ & 0.0015 & $(2.1)$ \\
$\sigma\left(\log \left[10^{10} A_{S}\right]\right)$ & 0.013 & 0.0085 & $(1.5)$ & 0.0055 & $(2.4)$ \\
$\sigma\left(p_{\text {ann }}\right)$ & $<1.5 \cdot 10^{-7}$ & $<1.2 \cdot 10^{-7}$ & $(1.2)$ & $<6.3 \cdot 10^{-8}$ & $(2.4)$ \\
\hline \hline
\end{tabular}

TABLE V. $68 \%$ c.l. errors on cosmological parameters in the case of dark matter annihilation. The upper limits on $p_{a n n}$ are at $95 \%$ c.l.. The parameter $p_{a n n}$ is measured in $\left[\mathrm{m}^{3} / \mathrm{s} / \mathrm{Kg}\right]$. The numbers in brackets show the improvement factor $\sigma_{\text {Planck }} / \sigma$ respect to the Planck experiment.

WMAP5 at almost $2-\sigma$ c.l.

Results reported in Table $\nabla$ will exclude these models at more than $\sim 10-\sigma$ c.l. for Planck and Plank + ACT and at $\sim 20-\sigma$ for $\mathrm{CMBPol}$, as shown in figure 7 .

It is also interesting to notice that the constraints obtained by CMBpol are comparable to those obtained by a cosmic variance limited (CVL) experiment with angular resolution comparable to Planck and without lensing extraction. In fact, such a CVL experiment gives a constraint of $p_{\text {ann }}=5 \times 10^{-8}$ [37], comparable to the one reported in Table $\nabla$ for CMBpol. Finally, it is worth noting that adding small scale data from ACT improves the constraints obtained with Planck only data by just $20 \%$.

\section{E. Future Constraints on Helium Abundance}

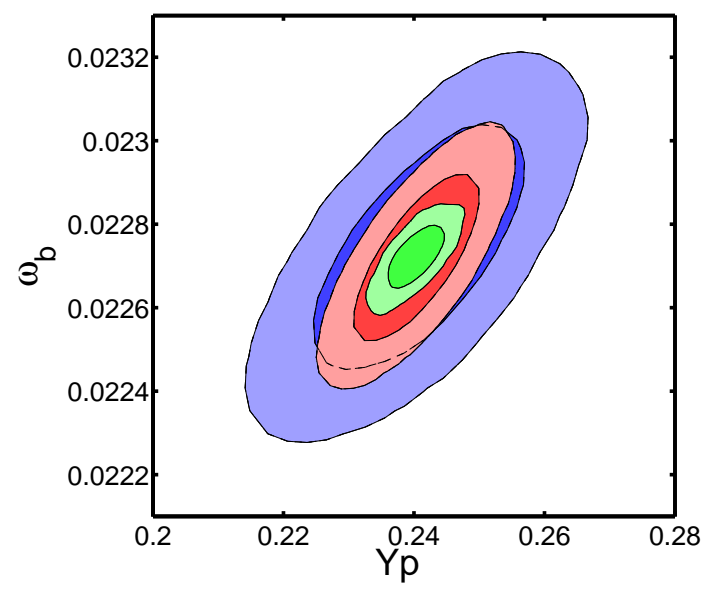

FIG. 8. $68 \%$ and $95 \%$ likelihood contour plots on the $Y_{H e}-\omega_{b}$ plane for Planck (blue), Planck+ACTPol (red) and CMBPol (green).

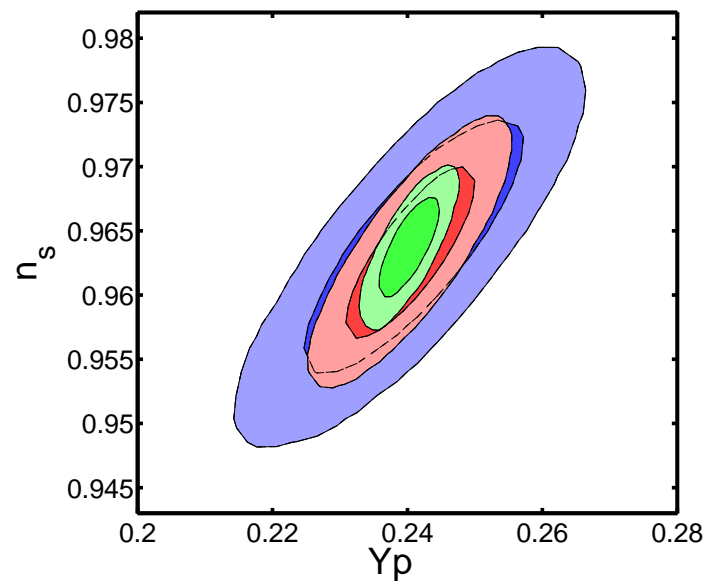

FIG. 9. $68 \%$ and $95 \%$ likelihood contour plots on the $Y_{H e}-n_{s}$ plane for Planck (blue), Planck+ACTPol (red) and CMBPol (green).

As recently shown by several authors ([46], [47], [48], 49]) the small scale CMB anisotropy spectrum can provide a powerful method for accurately determining the primordial ${ }^{4} \mathrm{He}$ abundance. Current astrophysical measurements of primordial fractional abundance $Y_{p}={ }^{4} \mathrm{He} /\left(\mathrm{H}+{ }^{4} \mathrm{He}\right)$ can be contained in the conservative estimate of $Y_{p}=0.250 \pm 0.003$ (see e.g. [50]).

As we can see from Table VI, the Planck satellite mission alone will not reach this accuracy, even when combined with ACT. However it is important to note that the Helium abundance in the BBN scenario is a growing function of $N_{\text {eff }}$ and the baryon density. A change in $\Delta N_{\text {eff }} \sim 1$ could produce a $\sim 5 \%$ variation in $Y_{p}$ that could be measurable by Planck or Planck+ACTPol. Moreover, a CMBPol-like experiment has the potential of reaching a precision comparable with current astrophysical measurements. This will open a new window of research for testing systematics in current primordial helium determinations.

\begin{tabular}{r|c|cc|cc}
\hline \hline $\begin{array}{r}\text { Parameter } \\
\text { uncertainty }\end{array}$ & Planck & \multicolumn{2}{|c|}{ Planck+ACTPol } & \multicolumn{2}{|c}{ CMBPol } \\
\hline$\sigma\left(\Omega_{b} h^{2}\right)$ & 0.00019 & 0.00013 & $(1.5)$ & 0.000051 & $(3.7)$ \\
$\sigma\left(\Omega_{c} h^{2}\right)$ & 0.0010 & 0.00064 & $(1.5)$ & 0.00027 & $(3.7)$ \\
$\sigma\left(\theta_{S}\right)$ & 0.00046 & 0.00026 & $(1.8)$ & 0.00010 & $(4.6)$ \\
$\sigma(\tau)$ & 0.0043 & 0.0035 & $(1.2)$ & 0.0023 & $(1.9)$ \\
$\sigma\left(n_{S}\right)$ & 0.0063 & 0.0042 & $(1.5)$ & 0.0025 & $(2.5)$ \\
$\sigma\left(\log \left[10^{10} A_{S}\right]\right)$ & 0.013 & 0.013 & $(1.0)$ & 0.0079 & $(1.6)$ \\
$\sigma\left(Y_{p}\right)$ & 0.010 & 0.0060 & $(1.6)$ & 0.0029 & $(3.4)$ \\
\hline \hline
\end{tabular}

TABLE VI. $68 \%$ c.l. errors on cosmological parameters in the case of helium abundance. The numbers in brackets show the improvement factor $\sigma_{\text {Planck }} / \sigma$ respect to the Planck experiment.

Comparing the results in TableVI with the constraints 
obtained in the case of a standard analysis in Table II it is easy to see that the major impact of including this parameter is on the determination of the scalar spectral index $n_{s}$ and the baryon abundance, with the 1- $\sigma$ c.l. increased by a factor $\sim 2$. In Figures 8 and 9 we plot the 2-D likelihood contours at $68 \%$ and $95 \%$ c.l. between $Y_{p}$ and these parameters.

\section{F. Future Constraints on Dark Energy Equation of State}

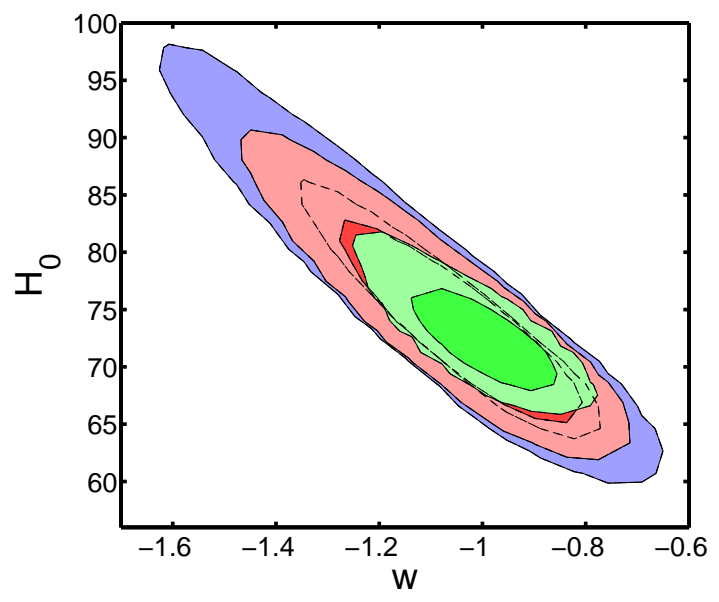

FIG. 10. $68 \%$ and $95 \%$ likelihood contour plots on the $w-H_{0}$ plane for Planck (blue), Planck+ACTPol (red) and CMBPol (green).

As is well known, primary CMB anisotropies are not able to provide accurate measurements of the dark energy equation of state because of geometrical degeneracies present with other parameters such as the amplitude of the dark energy density itself (see e.g. [51]) or the Hubble parameter. However, the inclusion of CMB lensing can break these degeneracies (see [52], 53]), as we can see in Table VII and from Figure [10. It is interesting to notice that the error on $\mathrm{w}$ is strongly dominated by the degenaracy with $H_{0}$. In fact, the constraints on $\mathrm{w}$ from the 3 datasets considered are almost the same if one adds a strong prior on $H_{0}$ at a level of $2 \%$, obtaining $\sigma(w)=0.039$ for Planck, $\sigma(w)=0.037$ for Planck $+\mathrm{ACT}$ and $\sigma(w)=0.033$ for CMBpol.

\section{G. Future Constraints on Variations of Fundamental Constants}

CMB anisotropies are sensitive to variations in fundamental constants such as the fine structure constant $\alpha$ (see e.g. [54], 55], [56], [57]) or Newton's constant $G([58]$ ) through changes in the recombination scenario. Varying $\alpha$ changes the ionization and excitation rates and can delay or accelerate recombination. Varying $G$ does not af-

\begin{tabular}{r|c|cc|cc}
\hline \hline $\begin{array}{r}\text { Parameter } \\
\text { uncertainty }\end{array}$ & Planck & \multicolumn{2}{|c|}{ Planck+ACTPol } & \multicolumn{2}{c}{ CMBPol } \\
\hline$\sigma\left(\Omega_{b} h^{2}\right)$ & 0.00013 & 0.000080 & $(1.6)$ & 0.000032 & $(4.2)$ \\
$\sigma\left(\Omega_{c} h^{2}\right)$ & 0.0011 & 0.00072 & $(1.5)$ & 0.00038 & $(3.0)$ \\
$\sigma\left(\theta_{s}\right)$ & 0.00026 & 0.00016 & $(1.6)$ & 0.000053 & $(4.9)$ \\
$\sigma(\tau)$ & 0.0040 & 0.0033 & $(1.2)$ & 0.0023 & $(1.8)$ \\
$\sigma\left(n_{s}\right)$ & 0.0032 & 0.0022 & $(1.4)$ & 0.0016 & $(2.0)$ \\
$\sigma\left(\log \left[10^{10} A s\right]\right)$ & 0.013 & 0.0098 & $(1.3)$ & 0.0070 & $(1.9)$ \\
$\sigma(w)$ & 0.2 & 0.15 & $(1.3)$ & 0.085 & $(2.4)$ \\
\hline \hline
\end{tabular}

TABLE VII. $68 \%$ c.l. errors on cosmological parameters from future surveys in case of a variable dark energy equation of state $w$. The numbers in brackets show the improvement factor $\sigma_{\text {Planck }} / \sigma$ respect to the Planck experiment.

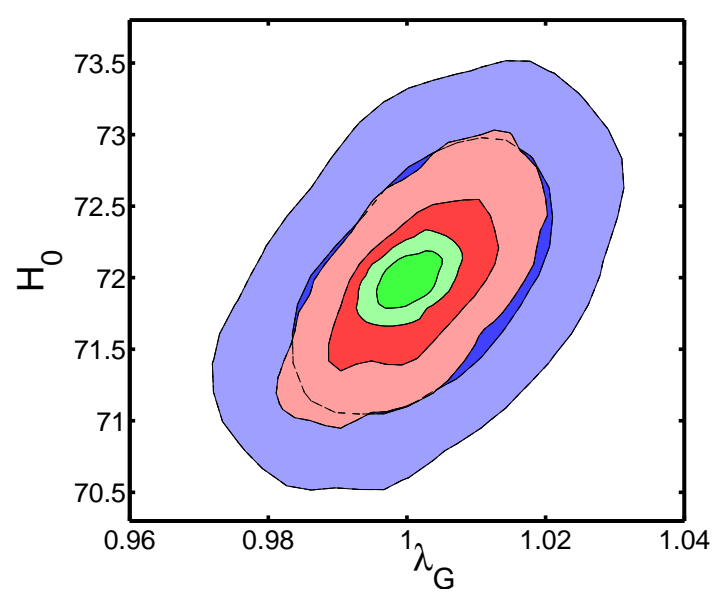

FIG. 11. $68 \%$ and $95 \%$ likelihood contour plots on the $\lambda_{G}-H_{0}$ plane for Planck (blue), Planck+ACTPol (red) and CMBPol (green).

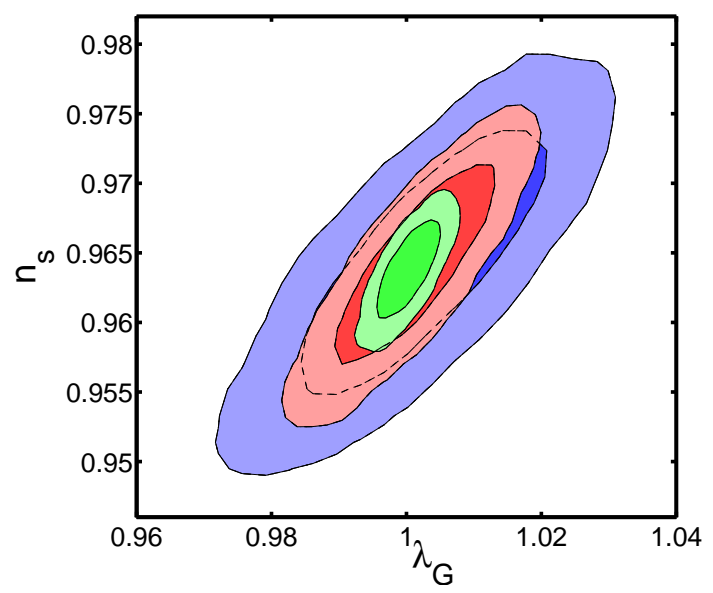

FIG. 12 . $68 \%$ and $95 \%$ likelihood contour plots on the $\lambda_{G}-n_{s}$ plane for Planck (blue), Planck+ACTPol (red) and CMBPol (green).

fect recombination directly but "rescales" the expansion 


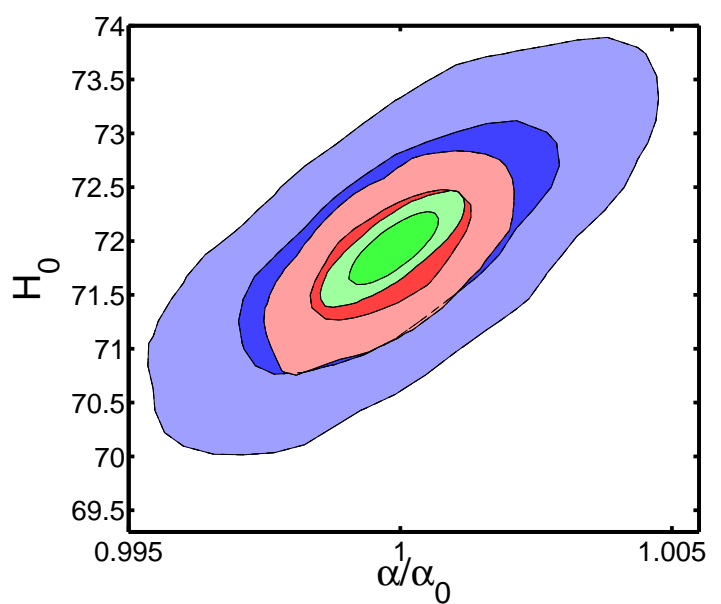

FIG. $13.68 \%$ and $95 \%$ likelihood contour plots on the $\alpha / \alpha_{0}$ - $H_{0}$ plane for Planck (blue), Planck+ACTPol (red) and CMBPol (green).

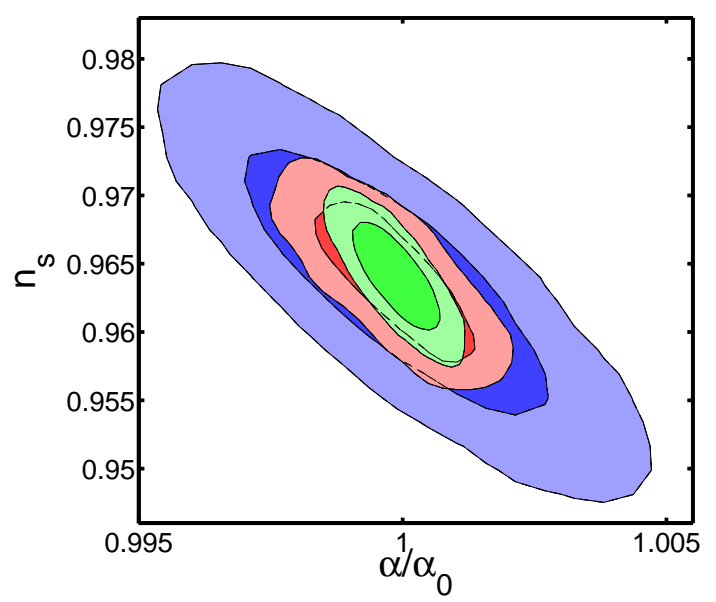

FIG. 14. $68 \%$ and $95 \%$ likelihood contour plots on the $\alpha / \alpha_{0}$ - $n_{s}$ plane for Planck (blue), Planck+ACTPol (red) and CMBPol (green).

rate of the Universe, changing the epoch when recombination takes place.

The constraints are reported in Table IX and Table VIII for variations in $\alpha$ and $G$ respectively. In order to parametrize the variations with dimensionless quantities we have considered variations in the parameters $\Delta_{\alpha}=$ $\alpha / \alpha_{0}$ e $\lambda_{G}=G / G_{0}$ where $\alpha_{0}$ and $G_{0}$ are the current values of these fundamental constants, measured in the laboratory $\alpha_{0}=7.2973525376(50) \times 10^{-3}$ and $G_{0}=$ $6.67428(67) \times 10^{-11} \mathrm{~m}^{3} \mathrm{~kg}^{-1} \mathrm{~s}^{-2}$.

As we can see from Tables IX and IVIII, a variation in these fundamental constants has important effects for the determination of the scalar spectral index $n_{s}$ and the Hubble costant $H_{0}$. This can also be seen in the 2-D

\footnotetext{
${ }^{3}$ See http://www.codata.org/
}

\begin{tabular}{r|c|cc|cc}
\hline \hline $\begin{array}{r}\text { Parameter } \\
\text { uncertainty }\end{array}$ & Planck & Planck+ACTPol & \multicolumn{2}{|c}{ CMBPol } \\
\hline$\sigma\left(\Omega_{b} h^{2}\right)$ & 0.00019 & 0.00013 & $(1.5)$ & 0.000048 & $(3.9)$ \\
$\sigma\left(\Omega_{c} h^{2}\right)$ & 0.0010 & 0.00068 & $(1.5)$ & 0.00025 & $(4.0)$ \\
$\sigma(\tau)$ & 0.0042 & 0.0037 & $(1.1)$ & 0.0022 & $(1.9)$ \\
$\sigma\left(H_{0}\right)$ & 0.60 & 0.40 & $(1.5)$ & 0.13 & $(4.6)$ \\
$\sigma\left(n_{s}\right)$ & 0.0061 & 0.0046 & $(1.3)$ & 0.0023 & $(2.6)$ \\
$\sigma\left(\log \left[10^{10} A_{s}\right]\right)$ & 0.018 & 0.013 & $(1.4)$ & 0.0073 & $(2.5)$ \\
$\sigma\left(\lambda_{G}\right)$ & 0.012 & 0.0076 & $(1.6)$ & 0.0030 & $(4.0)$ \\
\hline \hline
\end{tabular}

TABLE VIII. $68 \%$ c.l. errors on cosmological parameters from future surveys in case of a variable gravitational constant G. The numbers in brackets show the improvement factor $\sigma_{\text {Planck }} / \sigma$ respect to the Planck experiment.

\begin{tabular}{r|c|cc|cc}
\hline \hline $\begin{array}{r}\text { Parameter } \\
\text { uncertainty }\end{array}$ & Planck & Planck+ACTPol & \multicolumn{2}{|c}{ CMBPol } \\
\hline$\sigma\left(\Omega_{b} h^{2}\right)$ & 0.00013 & 0.000087 & $(1.6)$ & 0.000035 & $(4.1)$ \\
$\sigma\left(\Omega_{c} h^{2}\right)$ & 0.0012 & 0.00072 & $(1.7)$ & 0.00032 & $(3.9)$ \\
$\sigma(\tau)$ & 0.0042 & 0.0034 & $(1.2)$ & 0.0024 & $(1.8)$ \\
$\sigma\left(H_{0}\right)$ & 0.77 & 0.40 & $(1.9)$ & 0.21 & $(3.8)$ \\
$\sigma\left(n_{s}\right)$ & 0.0060 & 0.0036 & $(1.8)$ & 0.0026 & $(2.6)$ \\
$\sigma\left(\log \left[10^{10} A_{s}\right]\right)$ & 0.015 & 0.011 & $(1.4)$ & 0.0042 & $(2.5)$ \\
$\sigma\left(\alpha / \alpha_{0}\right)$ & 0.0018 & 0.00095 & $(2.0)$ & 0.00053 & $(3.7)$ \\
\hline \hline
\end{tabular}

TABLE IX. $68 \%$ c.l. errors on cosmological parameters from future surveys in case of a variable fine structure constant $\alpha$. The numbers in brackets show the improvement factor $\sigma_{\text {Planck }} / \sigma$ respect to the Planck experiment.

likelihood contour plots in Figures 11, 12, 13, and 14,

\section{CONCLUSIONS}

In this paper we have performed a systematic analysis of the future constraints on several parameters achievable from CMB experiments. Aside from the 5 parameters of the standard $\Lambda$-CDM model we have considered new parameters mostly related to quantities which can be probed in a complementary way in the laboratory and/or with astrophysical measurements. In particular we found that the Planck experiment will provide bounds on the sum of the masses $\Sigma m_{\nu}$ that could potentially definitively confirm or rule out the HeidelbergMoscow claim for a detection of an absolute neutrino mass scale. Planck+ACTPol could reach sufficient sensitivity for a robust detection of neutrino mass for an inverted hierarchy, while CMBPol should also be able to detect it for a direct mass hierarchy. The comparison of Planck+ACTPol constraints on baryon density, $N_{\text {eff }}$ and $Y_{p}$ with the complementary bounds from BBN will provide a fundamental test for the whole cosmological scenario. CMBPol could have a very important impact in understanding the epoch of neutrino decou- 
pling. Moreover, the primordial Helium abundance can be constrained with an accuracy equal to that of current astrophysical measurements but with much better control of systematics. Constraints on fundamental constants can be achieved at a level close to laboratory constraints. Such overlap between cosmology and other fields of physics and astronomy is one of the most interesting aspect of future CMB research.

We should note, however, that our forecasts rely on several technical assumptions. First, we assumed that the theoretical model of the recombination process is accurately known. This is not quite true as corrections to the recombination process are already needed for the Planck experiment (see e.g. [66]). However, this is mainly a computational problem that could be solved by the time of CMBPol launch, expected not before 2015.
In addition, we assume that the foreground and beam uncertainties are smaller than the statistical errors.

Nevertheless, the results clearly show the advantage of adding small scale data from the ACT telescope to the Planck satellite data. Adding the former to the latter will improve the constraints by a factor $\sim 2$ in most of the models considered.

\section{ACKNOWLEDGMENTS}

This research has been supported by ASI contract I/016/07/0 "COFIS." BS is supported by an NSF graduate fellowship. DNS is supported by NASA theory Grant No. NNX087AH30G and NSF Grant No. 0707731.
[1] G. F. Smoot et al., Astrophys. J. 396 (1992) L1; C. L. Bennett et al., Astrophys. J. 464 (1996) L1 arXiv:astro-ph/9601067.

[2] A. D. Miller et al., Astrophys. J. 524 (1999) L1 arXiv:astro-ph/9906421; A. Melchiorri et al. [Boomerang Collaboration], Astrophys. J. 536 (2000) L63 arXiv:astro-ph/9911445; P. de Bernardis et al. [Boomerang Collaboration], Nature 404 (2000) 955 arXiv:astro-ph/0004404; S. Hanany et al., Astrophys. J. $\mathbf{5 4 5}$ (2000) L5 arXiv:astro-ph/0005123; N. W. Halverson et al., Astrophys. J. 568 (2002) 38 arXiv:astro-ph/0104489; Reichardt, C. L., et al. 2009, Astrophys. J., 694, 1200 ; QUaD collaboration, et al. 2009, arXiv:0909.1621 Sievers, J. L., et al. 2009, arXiv:0901.4540.

[3] D. N. Spergel et al. [WMAP Collaboration], Astrophys. J. Suppl. 148 (2003) 175 arXiv:astro-ph/0302209; C. L. Bennett et al. [WMAP Collaboration], Astrophys. J. Suppl. 148 (2003) 1 arXiv:astro-ph/0302207; Komatsu, E., et al. 2009, Astrophys. J. Suppl., 180, 330; Komatsu, E., et al. 2010, arXiv:1001.4538.

[4] [Planck Collaboration], arXiv:astro-ph/0604069.

[5] M. J. White, Astrophys. J. 555 (2001) 88 arXiv:astro-ph/0101086; A. Cooray, arXiv:astro-ph/0203048.

[6] U. Seljak and M. Zaldarriaga, Phys. Rev. Lett. 78 (1997) 2054 arXiv:astro-ph/9609169; M. Kamionkowski, A. Kosowsky and A. Stebbins, Phys. Rev. D 55 (1997) 7368 arXiv:astro-ph/9611125.

[7] Dunkley, J., et al. 2009, American Institute of Physics Conference Series, 1141, 222

[8] M. Zaldarriaga and U. Seljak, Phys. Rev. D 58 (1998) 023003 arXiv:astro-ph/9803150; A. Lewis and A. Challinor, Phys. Rept. 429 (2006) 1 arXiv:astro-ph/0601594].

[9] [The ACT Collaboration], arXiv:1001.2934]

[10] M. Lueker et al., arXiv:0912.4317.

[11] P. Oxley et al., Proc. SPIE Int. Soc. Opt. Eng. 5543 (2004) 320 arXiv:astro-ph/0501111.

[12] B. P. Crill et al., arXiv:0807.1548 [astro-ph].

[13] J. E. Carlstrom et al., arXiv:0907.4445 [astro-ph.IM].

[14] A. Kosowsky [the ACT Collaboration], New Astron. Rev. 50 (2006) 969 arXiv:astro-ph/0608549.
[15] J. Bock et al. [CMBPol Collaboration], arXiv:0906.1188 [astro-ph.CO].

[16] P. de Bernardis, M. Bucher, C. Burigana, L. Piccirillo and f. P. Collaboration, Exper. Astron. 23 (2009) 5 arXiv:0808.1881 [astro-ph]].

[17] M. Shimon, N. J. Miller, C. T. Kishimoto, C. J. Smith, G. M. Fuller and B. G. Keating, arXiv:1001.5088 [astroph.CO].

[18] D. Baumann et al. [CMBPol Study Team Collaboration], AIP Conf. Proc. 1141 (2009) 10 arXiv:0811.3919 [astro$\mathrm{ph}]$.

[19] M. Zaldarriaga et al., arXiv:0811.3918 [astro-ph].

[20] S. Dodelson et al., arXiv:0902.3796 [astro-ph.CO].

[21] A. Lewis, A. Challinor and A. Lasenby, Astrophys. J. 538 (2000) 473 arXiv:astro-ph/9911177.

[22] C. M. Hirata, U. Seljak, Phys. Rev. D 68(2003) 083002 [arXiv:0306354 [astro-ph]];

[23] T. Okamoto, W. Hu, Phys.Rev. D 67 (2003) 083002 arXiv:astro-ph/0301031 2

[24] L. Perotto, J. Lesgourgues, S. Hannestad, H. Tu and Y. Y. Y. Wong, JCAP 0610 (2006) 013 arXiv:astro-ph/0606227.

[25] C. P. Ma and E. Bertschinger, Astrophys. J. 455 (1995) 7 arXiv:astro-ph/9506072].

[26] K. Ichikawa, M. Fukugita and M. Kawasaki, Phys. Rev. D 71 (2005) 043001 arXiv:astro-ph/0409768.

[27] A. Slosar, Phys. Rev. D 73, 123501 (2006) arXiv:astro-ph/0602133.

[28] M. Kaplinghat, L. Knox and Y. S. Song, Phys. Rev. Lett. 91 (2003) 241301 arXiv:astro-ph/0303344.

[29] G. L. Fogli et al., Phys. Rev. D 78 (2008) 033010 arXiv:0805.2517 [hep-ph]].

[30] Beck, M., \& the Katrin collaboration 2010, Journal of Physics Conference Series, 203, 012097

[31] J. R. Kristiansen and O. Elgaroy, JCAP 0801 (2008) 007 arXiv:0709.4152 [astro-ph]].

[32] H. V. Klapdor-Kleingrothaus, I. V. Krivosheina, A. Dietz and O. Chkvorets, Phys. Lett. B 586 (2004) 198 arXiv:hep-ph/0404088.

[33] Monfardini, A., et al. 2006, Nuclear Instruments and Methods in Physics Research A, 559, 346 
[34] R. Bowen, S. H. Hansen, A. Melchiorri, J. Silk and R. Trotta, Mon. Not. Roy. Astron. Soc. 334, 760 (2002) arXiv:astro-ph/0110636.

[35] G. Mangano, G. Miele, S. Pastor and M. Peloso, Phys. Lett. B 534 (2002) 8 arXiv:astro-ph/0111408.

[36] G. Mangano, G. Miele, S. Pastor, T. Pinto, O. Pisanti and P. D. Serpico, Nucl. Phys. B 756 (2006) 100 arXiv:hep-ph/0607267.

[37] Galli, S., Iocco, F., Bertone, G., \& Melchiorri, A. 2009, Phys. Rev. D, 80, 023505;

[38] L. Zhang, X. L. Chen, Y. A. Lei and Z. G. Si, Phys. Rev. D 74, 103519 (2006) arXiv:astro-ph/0603425; L. Zhang, X. Chen, M. Kamionkowski, Z. g. Si and Z. Zheng, Phys. Rev. D 76, 061301 (2007) arXiv:0704.2444 [astro-ph]].

[39] Chen, X., \& Kamionkowski, M. 2004, Phys. Rev. D, 70, 043502

[40] Slatyer, T. R., Padmanabhan, N., \& Finkbeiner, D. P. 2009, Phys. Rev. D, 80, 043526

[41] Adriani, O., et al. 2009, Nature (London), 458, 607

[42] Chang, J., et al. 2008, Nature (London), 456, 362

[43] Latronico, L., \& for the Fermi LAT Collaboration 2009, arXiv:0907.0452

[44] Cholis, I., Dobler, G., Finkbeiner, D. P., Goodenough, L., \& Weiner, N. 2009, Phys. Rev. D, 80, 123518

[45] Grajek, P., Kane, G. L.,Phalen, D. J., Pierce, A., \& Watson, S. 2009, Phys. Rev. D, 79, 043506

[46] R. Trotta and S. H. Hansen, Phys. Rev. D 69, 023509 (2004) arXiv:astro-ph/0306588.

[47] K. Ichikawa, T. Sekiguchi and T. Takahashi, Phys. Rev. D 78 (2008) 043509 arXiv:0712.4327 [astro-ph]].

[48] Hamann, J., Lesgourgues, J., \& Mangano, G. 2008, Journal of Cosmology and Astro-Particle Physics, 3, 4

[49] Komatsu, E., et al., 2010, arXiv:1001.4538

[50] F. Iocco, G. Mangano, G. Miele, O. Pisanti and P. D. Serpico, Phys. Rept. 472, 1 (2009) arXiv:0809.0631 [astro$\mathrm{ph}]$.
[51] R. Bean and A. Melchiorri, Phys. Rev. D 65, 041302 (2002) arXiv:astro-ph/0110472.

[52] W. Hu, Phys. Rev. D $65 \quad$ (2002) 023003 arXiv:astro-ph/0108090.

[53] Efstathiou, G., \& Bond, J. R. 1999, Mon. Not. R. Astron. Soc., 304, 75

[54] Menegoni, E., Galli, S., Bartlett, J. G., Martins, C. J. A. P., \& Melchiorri, A. 2009, Phys. Rev. D, 80, 087302

[55] Martins, C. J. A. P., Menegoni, E., Galli, S., Mangano, G., \& Melchiorri, A. 2010, arXiv:1001.3418

[56] Ichikawa, K., Kanzaki, T., \& Kawasaki, M. 2006, Phys. Rev. D, 74, 023515

[57] Landau, S. J., \& Scóccola, C. G. 2010, arXiv:1002.1603

[58] Galli, S., Melchiorri, A., Smoot, G. F., \& Zahn, O. 2009, Phys. Rev. D, 80, 023508 O. Zahn and M. Zaldarriaga, Phys. Rev. D 67 (2003) 063002 arXiv:astro-ph/0212360.

[59] Zahn, O., \& Zaldarriaga, M. 2003, Phys. Rev. D, 67, 063002

[60] E. Komatsu et al. [WMAP Collaboration], Astrophys. J. Suppl. 180 (2009) 330 arXiv:0803.0547 [astro-ph]]; J. Dunkley et al. [WMAP Collaboration], arXiv:0803.0586 [astro-ph].

[61] C. J. MacTavish et al., Astrophys. J. 647 (2006) 799 arXiv:astro-ph/0507503; F. Piacentini et al., Astrophys. J. 647 (2006) 833 arXiv:astro-ph/0507507; T. E. Montroy et al., Astrophys. J. 647 (2006) 813 arXiv:astro-ph/0507514; Jones, W. C., et al. 2006, Astrophys. J. 647, 823

[62] C. L. Reichardt et al., arXiv:0801.1491 [astro-ph].

[63] P. G. Castro et al. [QUaD collaboration], arXiv:0901.0810 [astro-ph.CO].

[64] J. Bock et al., arXiv:0805.4207 [astro-ph].

[65] A. Lewis and S. Bridle, Phys. Rev. D 66 (2002) 103511 arXiv:astro-ph/0205436.

[66] J. A. Rubino-Martin, J. Chluba, W. A. Fendt and B. D. Wandelt, arXiv:0910.4383 [astro-ph.CO]. 